\title{
The Deactivation Mechanism of $\mathrm{RuO}_{2}-\mathrm{IrO}_{2}-\mathrm{SnO}_{2} / \mathrm{Ti}$ Anodes Under Alternative Current Electrolysis Condition
}

\author{
Yonglei Xin ${ }^{\mathrm{a}}$, Likun $\mathrm{Xu}^{\mathrm{b}}$ and Juntao Wang ${ }^{\mathrm{c}}$ \\ Science and Technology on Marine Corrosion and Protection Laboratory, \\ Luoyang Ship Material Research Institute, Qingdao, China, 266101 \\ axinyonglei@163.com, bxulk@sunrui.net, cwangjt@sunrui.net
}

Keywords: $\mathrm{RuO}_{2}-\mathrm{IrO}_{2}-\mathrm{SnO}_{2} / \mathrm{Ti}$, Alternative Current Electrolysis, Deactivation Mechanism, Pechini method.

\begin{abstract}
The $\mathrm{RuO}_{2}-\mathrm{IrO}_{2}-\mathrm{SnO}_{2} / \mathrm{Ti}$ anodes were prepared by the sol-gel procedure. SEM, EDX and XRD showed that the rutile structured $\mathrm{RuO}_{2}-\mathrm{IrO}_{2}-\mathrm{SnO}_{2}$ solid solution was formed after pyrolysis, the solid solution structure and active components were almost disappeared after the deactivation of the anodes. Electrochemical results indicated that the active area decreased and the coating resistance increased, which are resulted by active component falling off from the anode surfaces. Hence, the deactivation of the oxide anodes was due to detachment of the active component under alternative current electrolysis.
\end{abstract}

\section{Introduction}

The anti-fouling technology of seawater electrolysis is useful to prevent marine fouling in the seawater pipeline system. Metal oxide coated titanium anode is extensively used in seawater electrolysis due to its good performance over the traditional anodes such as graphite and lead-silver alloy ${ }^{[1]}$. Electrocatalysts $\mathrm{RuO}_{2}-\mathrm{IrO}_{2}-\mathrm{SnO}_{2}$ demonstrates high activity together with a considerably higher stability in chlorine evolution reaction in comparison with $\mathrm{RuO}_{2}-\mathrm{TiO}_{2}$ or $\mathrm{RuO}_{2}-\mathrm{SnO}_{2}$ anode. This leads to the development of mixed $\mathrm{RuO}_{2}-\mathrm{IrO}_{2}-\mathrm{SnO}_{2}$ coating. $\mathrm{RuO}_{2}-\mathrm{IrO}_{2}-\mathrm{SnO}_{2}$ coated titanium electrodes are now becoming more and more attractive in seawater electrolysis industry as dimensionally stable anodes ${ }^{[2-3]}$. However, the lifetime of the electrode was drastically shortened during electrolysis with periodically reversed (PR) current compared to that in continuous anodic electrolysis ${ }^{[4]}$. Although our previous studies have revealed the deactivation mechanism of the oxide anode in continuous anodic electrolysis ${ }^{[5-7]}$, the deterioration mechanism induced by periodic cathodizing has been unknown. The aim of this study is to investigate the deactivation process of $\mathrm{RuO}_{2}-\mathrm{IrO}_{2}-\mathrm{SnO}_{2} / \mathrm{Ti}$ anodes under alternative current electrolysis condition.

\section{Experiment}

\subsection{Anode Preparation}

The titanium plate of Grade TA2 was utilized as the electrode substrate, which was degreased in acetone and then etched in the boiling 10\% oxalic acid for $2 \mathrm{~h}$ to produce gray surface with uniform roughness. The precursor solution was prepared through dissolving citric acid (CA) in ethylene glycol (EG) at about $60^{\circ} \mathrm{C}$ at first, and then in which $\mathrm{RuCl} 3, \mathrm{H} 2 \mathrm{IrCl6}$ and $\mathrm{SnCl} 4$ salt were added while stirring to form a coating paint with $\mathrm{Ru}$ : $\mathrm{Ir}: \mathrm{Sn}=17: 23: 60$ in mole ratio. The precursor solution was painted over the pretreated titanium substrate with a brush, which was dried at $120^{\circ} \mathrm{C}$ for $10 \mathrm{~min}$ in the oven, and then sintered in the furnace at $470^{\circ} \mathrm{C}$ for $10 \mathrm{~min}$. The above steps were repeated several times to get an oxide loading of about $6 \mathrm{~g} \mathrm{~m}-2$. The last baking was kept at the firing temperature for $1 \mathrm{~h}$.

\subsection{Anode characterization}

The microstructure of the oxide electrode was characterized with a Philip XL30 type SEM equipped with EDAX micro-analyzer. The operating voltage of electronic beam was $20 \mathrm{keV}$. XRD 


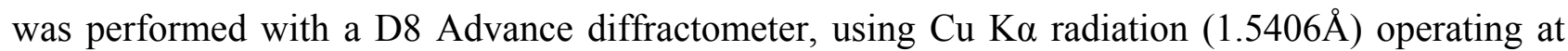
$40 \mathrm{kV}$ and $40 \mathrm{~mA}$.

2.3 Electrochemical measurement

All of the electrochemical measurements were performed using IM6 electrochemical workstation, in the electrolyte of $1 \mathrm{~mol} \mathrm{dm}-3 \mathrm{H} 2 \mathrm{SO} 4$ solution with platinum foil as counter electrode and saturated calomel electrode (SCE) as the reference electrode. The tests were made usually at room temperature except for the accelerated life test where the solution temperature was controlled at $40 \pm 1{ }^{\circ} \mathrm{C}$ with a water thermostat. In the alternative current electrolysis, the anodic current density was maintained at 2000A m-2, while the cathodic current density was 500A m-2, and alternative cycle frequency: 1 min. The electrolysis was interrupted periodically to make CV measurement. The CV curves were recorded in a range between $0.16 \mathrm{~V}$ and $1.16 \mathrm{~V}$ vs. SCE at a sweep rate of $20 \mathrm{mVs}-1$ after 10 cycles. The voltammetric charge was obtained by graphical integration of the anodic part of the voltammetric curve.

\section{Results and discussion}

\subsection{XRD}

Fig. 1 shows the XRD patterns of the $\mathrm{RuO} 2-\mathrm{IrO} 2-\mathrm{SnO} 2$ anode at different time of electrolysis. As shown in the figure, there are the broad peaks of $(\mathrm{Ru}, \mathrm{Sn}, \mathrm{Ir}) \mathrm{O} 2$ and the intense peaks of Ti substrate in the XRD of the non-electrolyzed anode. With the increase of electrolysis time, the intensity of diffraction peaks of ( $\mathrm{Ru}, \mathrm{Sn}, \mathrm{Ir}) \mathrm{O} 2$ gradually descend, but the diffraction angle has not been a big change. After degradation (1.5h), the intensity of diffraction peaks of (Ru, Sn, Ir)O2 almost disappear, which indicates that the oxide coating apparently break off on the inactive anode surface. However, the rutile $\mathrm{TiO} 2$ reflect is detected due to oxidization of Ti-based surface. With the electrolysis continuing, the anodically grown $\mathrm{TiO} 2$ films originally formed is thickened, and those sites from which catalyst layer leaves by mechanical loss starts to be anodically oxidized at the same time. The development of such a process leads to final degradation of anodes. In addition, none of the other new phases is observed.

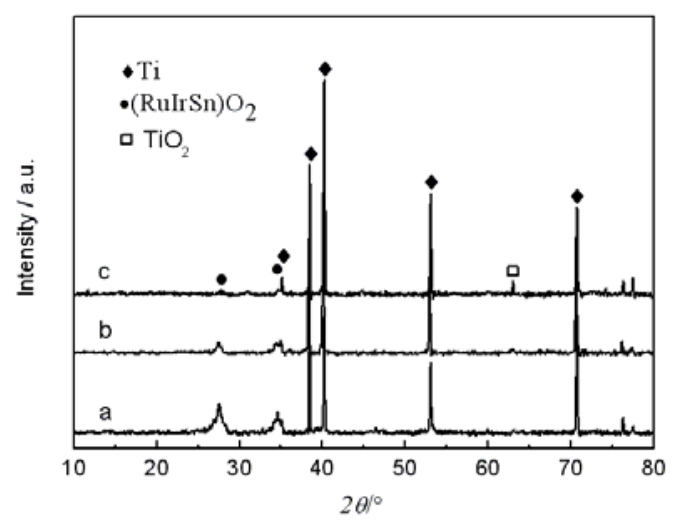

Fig. 1 XRD patterns of $\mathrm{RuO}_{2}-\mathrm{IrO}_{2}-\mathrm{SnO}_{2}$ anode at different time of electrolysis.

(a) non-electrolysis, (b) $0.6 \mathrm{~h}$, (c) after degradation

\subsection{SEM}

The morphologies of $\mathrm{RuO} 2-\mathrm{IrO} 2-\mathrm{SnO} 2$ anode at different time of electrolysis are shown in Fig. 2. The surface of the fresh anode consists of dried-mud cracks surrounded by compact areas, which tends to connect to form networks. This morphology of $\mathrm{RuO} 2-\mathrm{IrO} 2-\mathrm{SnO} 2$ anode is consistent with that in the literature $[3,8]$. Fig. 2(c) is surface micrography of the inactive RuO2-IrO2-SnO2 anode. As shown in the figure, some dried-mud cracks can not be found in some flat area, and the coating surface presents the rough morphology of the titanium substrate after acid etching, which is quite different from the surface morphologies after continuous anodic electrolysis. EDX indicates that intense Ti signal is present due to the exposure of the substrate, and a little amount of Ru, Ir and Sn is also probed in this area, moreover, it can be known from the above analysis of XRD that the inactive 
anode surface only remains a little amount of active components. Hence, the deactivation of the oxide anodes was due to detachment of the active component under alternative current electrolysis.
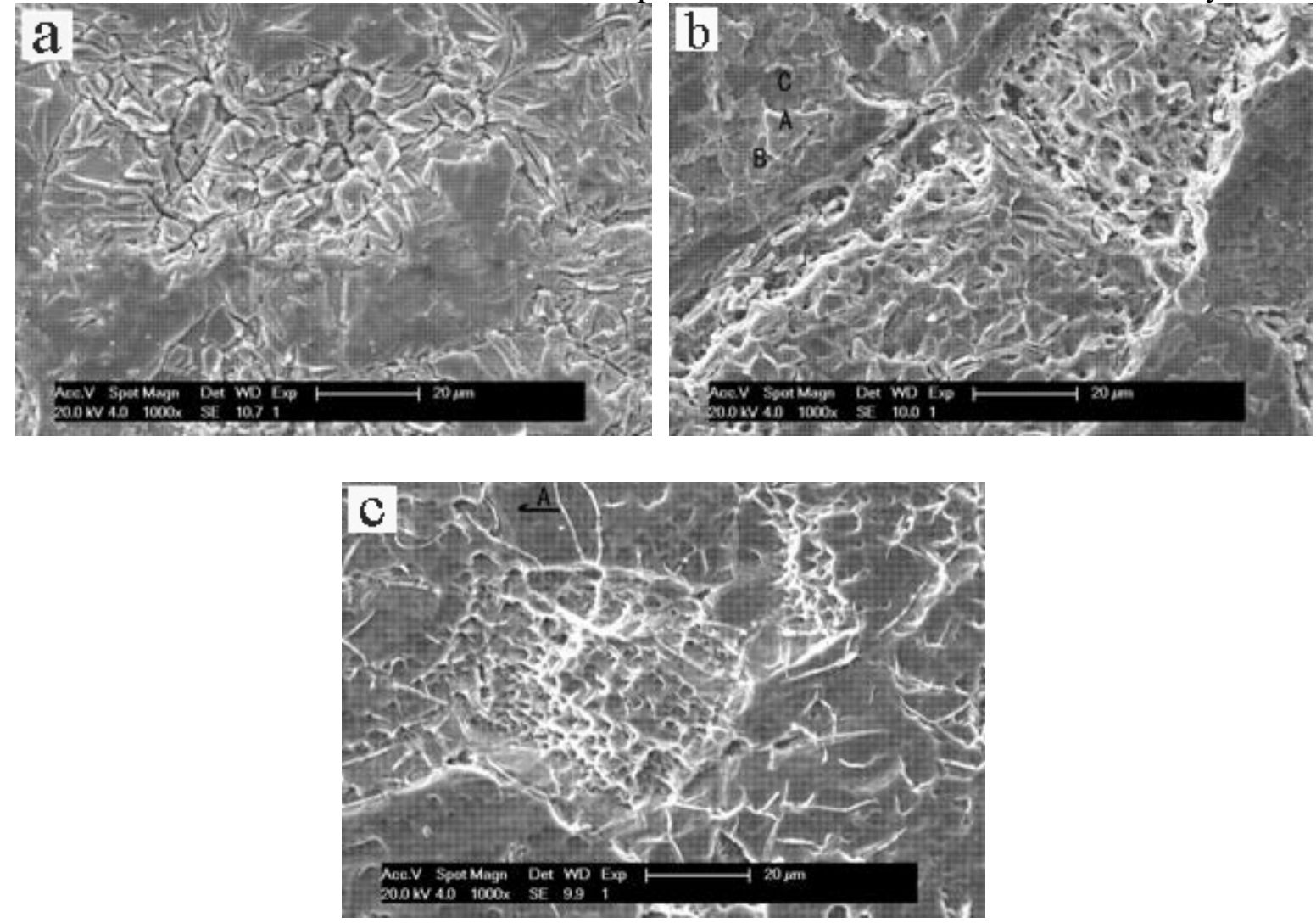

Fig. 2 Surface morphologies of $\mathrm{RuO}_{2}-\mathrm{IrO}_{2}-\mathrm{SnO}_{2}$ anode at different time of electrolysis.

(a) non-electrolysis, (b) $0.6 \mathrm{~h}$, (c) after degradation

\subsection{Cyclic voltammetry}

Fig. 3 shows the voltammetric curves of $\mathrm{RuO} 2-\mathrm{IrO} 2-\mathrm{SnO} 2$ anode in 1moldm-3 H2SO4 solution at different time of electrolysis. With the increase of electrolysis time, the voltammetric curve shows similar shape but lower current density. The voltammetric charge $\mathrm{q}^{*}$ obtained by the integration of the $\mathrm{CV}$ curve between oxygen and hydrogen evolution has been proven to be proportional to the number of electro-active sites, consequently, being able to characterize the electrochemically active surface area (EASA) of the oxide electrode [9]. The $\mathrm{q}^{*}$ values for the electrode (a), (b) and (c) are $11.25 \mathrm{mC} \cdot \mathrm{cm}^{-2}, 8.54 \mathrm{mC} \cdot \mathrm{cm}^{-2}$ and $3.34 \mathrm{mC} \cdot \mathrm{cm}^{-2}$, respectively, indicating $\mathrm{q}^{*}$ decreases with the electrolysis going on, and then falls steeply to the end. It can be deduced that electrocatalytic activity of oxide catalyst decreases with electrolysis time, which is resulted by active component falling off from the anode surface.

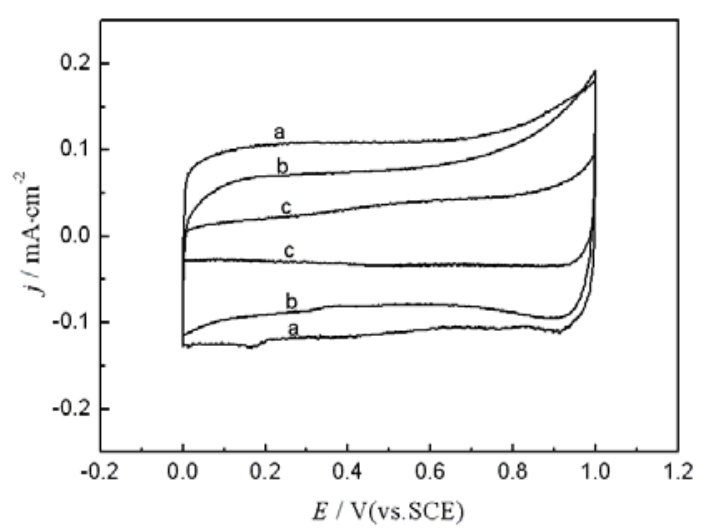

Fig. 3 Voltammetric curves of $\mathrm{RuO}_{2}-\mathrm{IrO}_{2}-\mathrm{SnO}_{2}$ anode at different time of electrolysis.

(a) non-electrolysis, (b) $0.6 \mathrm{~h}$, (c) after degradation 


\section{Summary}

According to the results given by $\mathrm{XRD}$, SEM and $\mathrm{CV}$ under alternative current electrolysis condition, the deactivation of $\mathrm{RuO} 2-\mathrm{IrO} 2-\mathrm{SnO} 2$ anode can be described as below. Electrocatalytic activity of oxide catalyst decreases with electrolysis time, which is resulted by active component falling off from the anode surface. Otherwise, the inactive anode surface only remains a little amount of $\mathrm{Ru}, \mathrm{Ir}$ and $\mathrm{Sn}$ components. Hence, the degradation mechanism of $\mathrm{RuO} 2-\mathrm{IrO} 2-\mathrm{SnO} 2$ anode under alternative current electrolysis condition differed from the oxide anode in continuous anodic electrolysis. The detachment of $\mathrm{RuO} 2-\mathrm{IrO} 2-\mathrm{SnO} 2$ anode coating is the main reason for electrode degradation in acid medium, however, two main degradation mechanisms of oxide anodes in continuous anodic electrolysis are as follows: (a) The chemical dissolution of anode coating in the medium; (b) The electrolyte permeated into the Ti/oxide interface, which led to an insulating TiO2 film generated on the Ti-based surface during the process of electrolysis.

The obtained results indicate that the incomplete utilization of the active mass of the anode coating is due to the loss of the coating activity caused by active component falling off from the anode surface. Therefore, we assume that introducing such a highly corrosion-resistant component as platinum and its oxides into the coating can enhance the stability of the oxide anode under alternative current electrolysis condition.

\section{References}

[1] C. H. Liang, N. B. Huang, Research on electrochemical behavior of Ti-Ir-Ru anode coating in electrolytic antifouling of flowing brine, J. Mater. Eng. Perform. 18 (2009) 1086-1090.

[2] M. E. Makgae, C. C. Theron and W. J. Przybylowicz, Preparation and surface characterization of $\mathrm{Ti} / \mathrm{SnO}_{2}-\mathrm{RuO}_{2}-\mathrm{IrO}_{2}$ thin films as electrode material for the oxidation of Phenol, Mater. Chem. Phys. 92 (2005) 559-564.

[3] L. Vazquez-Gomez, S. Ferro and A. De Battisti, Preparation and Characterization of $\mathrm{RuO}_{2}-\mathrm{IrO}_{2}-\mathrm{SnO}_{2}$ Ternary Mixtures for Advanced Electrochemical Technology, Appl. Catal., B 67(2006) 34-40.

[4] M. Morimitsu, R. Otogawa and M. Matsunaga, Effects of cathodizing on the morphology and composition of $\mathrm{IrO}_{2}-\mathrm{Ta}_{2} \mathrm{O}_{5} / \mathrm{Ti}$ anodes, Electrochim. Acta. 46 (2000) 401-406.

[5] L. K. Xu, J. D. Scantlebury, A study on the deactivation of an $\mathrm{IrO}_{2}-\mathrm{Ta}_{2} \mathrm{O}_{5}$ coated titanium anode Corros. Sci. 45 (2003) 2729-2740.

[6] J. M. Hu, H. M. Meng and J. Q. Zhang, Degradation mechanism of long service life $\mathrm{Ti} / \mathrm{IrO}_{2}-\mathrm{Ta}_{2} \mathrm{O}_{5}$ oxide anodes in sulphuric acid, Corros. Sci. 44 (2002) 1655-1668.

[7] V. I. Eberil, E. A. Novikov and A. F. Mazanko, Reasons for the DSA passivation during chlorate electrolysis and the means for extending the anode service life, Russ. J. Electrochem. 37 (2001) 1218-1222.

[8] V.V. Panic, A. Dekanski and S.K. Milonjic, $\mathrm{RuO}_{2}-\mathrm{TiO}_{2}$ coated titanium anodes obtained by the sol-gel procedure and their electrochemical behaviour in the chlorine evolution reaction Colloids Surf., A 157 (1999) 269-274.

[9] S. Trasatti, Physical electrochemistry of ceramic oxides, Electrochim. Acta. 36 (1991) 225-241. 